\title{
Género, violencia y riesgo en el tránsito. La dominación masculina en los discursos de automovilistas de la ciudad de Buenos Aires*
}

\section{| ${ }^{1}$ Rosa N. Geldstein, ${ }^{2}$ Pablo Francisco Di Leo, ${ }^{3}$ Silvina Ramos Margarido |}

Resumo: Presentamos resultados parciales de dos estudios multicéntricos sobre aspectos socioculturales y demográficos de los accidentes de tránsito en Argentina, conducidos entre 2004 y 2008 con el apoyo del Ministerio de Salud de la Nación. Nos centramos en las vinculaciones entre dimensiones de género y habitus de conducción que emergieron de 92 entrevistas en profundidad a automovilistas particulares de ambos sexos en la Ciudad Autónoma de Buenos Aires. El análisis cualitativo fue de tipo interpretativo y aplicó procedimientos de la teoría fundamentada (Grounded Theory). Utilizando la comparación constante, identificamos el fenómeno de la dominación masculina en los habitus y doxa (Bourdieu) de diferentes categorías de conductores en el campo social del tránsito. Las críticas hacia los estilos femeninos de conducción y la discriminación contra las mujeres se encontraron en los discursos dominantes de varones jóvenes y también en discursos femeninos, sugiriendo la eficacia de la dominación (masculina) simbólica. Conductoras jóvenes y adultas valoran positivamente el "pragmatismo masculino" en el manejo. Mientras las mujeres maduras naturalizan una supuesta superioridad masculina en la conducción, las jóvenes adoptan el pragmatismo en sus prácticas de manejo como mecanismo de inclusión legítima en ese campo. Pero tal pragmatismo implica prácticas reñidas con el respeto a las reglas y a los otros en el tránsito y se podría predecir que las condiciones de riesgo se incrementarán, a menos que se implementen políticas destinadas a fomentar un estilo de manejo responsable, aludido en un discurso sub-dominante de mujeres maduras que nos remite al concepto de ética del cuidado (Gilligan).

\author{
${ }^{1}$ Magíster en Estudios Sociales \\ de la Población (FLACSO- \\ CELADE), Investigadora \\ Independiente del CONICET \\ con sede en el Centro de \\ Estudios de Población - \\ CENEP. Correo electrónico: \\ rgeldstein@cenep.org.ar \\ 2 Doctor de la Universidad \\ de Buenos Aires en Ciencias \\ Sociales, Investigador del \\ CONICET con sede en el \\ Instituto de Investigaciones \\ Gino Germani, UBA. Correo \\ electrónico: pfdileo@gmail.com \\ ${ }^{3}$ Licenciada en Sociología (UBA), \\ Especialista en Organizaciones \\ No Gubernamentales (CEDES- \\ UdeSa-DiTella), Facultad de \\ Ciencias Sociales, UBA. Correo \\ electrónico: silvinarm@gmail.com
}

> Palavras-chave: accidentes de tránsito; violencia de género; automovilistas. 


\section{Introducción}

A partir de datos oficiales para el año 2000 se ha estimado que la población de Argentina pierde 170 mil años de vida potencial cada año debido a las defunciones por accidentes de tránsito (AT); la pérdida de esperanza de vida es de 0,43 años para varones y de 0,15 años para mujeres (LACASTA, 2006). ${ }^{1}$

En un análisis más reciente de la mortalidad por AT según sexo y edad por períodos quinquenales entre 1990 y 2004 y para el bienio 2005-2006, se confirma la continuidad del mayor riesgo de la población masculina, al señalar que durante esos años las muertes de varones se mantuvieron en alrededor del 75 por ciento del total de las defunciones por AT ocurridas en este país. Sin embargo, hay indicios de que la brecha entre la mortalidad masculina y la femenina por esta causa se podría estar achicando, debido al mayor incremento en el número de defunciones de mujeres. En efecto, entre 1990 y 2004 el número medio anual de defunciones por AT se incrementó de 3.500 a 3.800. Ello representa un aumento promedio del 8 por ciento, superado por el incremento de las muertes femeninas por esta causa, que alcanzó al 11,1 por ciento en esos mismos años. Más aún, cuando el número medio anual de muertes por AT, que había descendido en el quinquenio 2000-04, se volvió a incrementar durante el bienio 2005-2006 (9,8 por ciento respecto del quinquenio anterior), lo hizo especialmente entre las mujeres, cuyo incremento porcentual alcanzó al 16 por ciento (datos de ALTIERI, 2008).

Por otra parte, el riesgo de morir como consecuencia de un hecho de tránsito es desproporcionadamente mayor entre los jóvenes:

Mientras para otros grupos de edad la mortalidad por tumores o enfermedades del sistema circulatorio son las más frecuentes, la mortalidad por accidentes de tránsito representa junto con el resto de las causas externas, la principal causa de muerte entre los jóvenes entre 15 y 29 años (FISA, 2007). En 1990-94 los AT representaron el 14,4\% de las muertes de varones jóvenes y el 8,7\% de las mujeres jóvenes. En 2005-06 estos porcentajes se habían incrementado levemente, un poco más entre la mujeres.

La proporción de muertes por AT sobre el total de defunciones en los jóvenes era 10 veces superior que en la población total, con mayor diferencial entre las mujeres (15 veces) que en los varones (ALTIERI, 1998, p. 36-37)

Es así que, mientras las muertes de varones y mujeres entre los 15 y los 29 años no alcanzan a representar, respectivamente, el 5 y el 3 por ciento del total de defunciones masculinas y femeninas, dan cuenta de alrededor del 30 por ciento del total de muertes por AT, con escasa diferencia entre los sexos: 
Las muertes por accidentes de tránsito tuvieron, al igual que en el resto de las cau-

sas externas, mayor impacto sobre los jóvenes que sobre el total de la población. En 1990-94 se había registrado un promedio anual de 960 muertes de jóvenes, el 27\% de las defunciones totales por AT. En 2005-06, el promedio anual de defunciones de jóvenes había ascendido a 1160, y representaba el 30\% de las defunciones totales (ALTIERI, 2008, p. 36). ${ }^{2}$

Aunque todavía débiles, estas evidencias y una mirada atenta a los resultados previos de nuestras indagaciones cualitativas (GELDSTEIN, 2006; PETERLINI; GELDSTEIN, 2005) - así como la reflexión sobre nuestras propias experiencias como agentes sociales en el campo del tránsito - , nos llevaron a preguntarnos sobre el papel del género en el riesgo vial y sobre los mecanismos de reproducción social por los cuales las prácticas de riesgo, la discriminación y las actitudes de violencia interpersonal (fáctica y simbólica) se transmitirían a las nuevas generaciones de conductores. ${ }^{3}$

En tanto fenómeno multidimensional, los AT, sus consecuencias y sus posibles causas, han concitado la atención de investigadores de diversas disciplinas (con predominio de las ciencias médicas y la psicología) y han dado lugar a la utilización de diferentes estrategias metodológicas y técnicas, predominantemente cuantitativas. En una revisión reciente (GELDSTEIN; BERTONCELLO, 2006, p. 14-16), se detectó una amplia mayoría de estudios cuantitativos de tipo epidemiológico, que enfatizan el análisis de factores objetivos de efectos mensurables; con frecuencia su foco está puesto en el factor humano usuario de las vías como principal factor de riesgo. Dichos estudios, concentrados principalmente en EE.UU. y Europa, pero también existentes en Argentina, señalan sistemáticamente la incidencia de factores de riesgo como la presencia de alcohol, el exceso de velocidad, las malas condiciones climáticas, las franjas horarias, etc. Resulta imposible reseñar aquí sus resultados o citarlos sin incurrir en injustas omisiones, pero el lector interesado puede consultar, entre otras, la bibliografía citada en los distintos capítulos de la obra coordinada por Geldstein y Bertoncello (2006) y también los muy completos informes de organizaciones internacionales, como la Organización Mundial de la Salud (OMS), la Organización Panamericana de la Salud (OPS) o el Banco Mundial, que recogen resultados de diversas investigaciones, clásicas y recientes, a lo largo del mundo (OMS, 2002; 2004; OPS, 2004; GLIZER, 1993, citados en GELDSTEIN; BERTONCELLO, 2006). 
Aunque el peso de cada subpoblación recortada según criterios de sexo, edad, rol en el tránsito, tipo de vehículo, etc., varía bastante según los contextos, existe coincidencia entre los hallazgos - como los citados arriba - en cuanto a la concentración de víctimas (y también presuntamente de causantes) entre los varones jóvenes, de quienes se conocen o a quienes se atribuyen los comportamientos de riesgo más frecuentes y extremos. Sin embargo, es menos usual encontrar resultados de investigaciones empíricas que hayan indagado sobre las causas o los motivos de tales comportamientos y que apliquen estrategias comparativas. Más aún, en muchos casos (como en el de Argentina) las tasas de mortalidad por sexo y edad se calculan sobre la población total respectiva, por carecer de datos sobre el denominador real de la población expuesta a riesgo (por ejemplo, el número de hombres que conducen en relación al número de mujeres que lo hacen; la frecuencia de manejo y el kilometraje recorrido, etc.) En los países centrales de América y Europa es abundante la investigación cuantitativa sobre diversos aspectos relacionados con las actitudes y comportamientos de la población en el tránsito, los efectos de campañas de seguridad vial o los factores demográficos asociados al no uso de elementos de seguridad. Muchos estudios utilizan fuentes secundarias y, debido a la disponibilidad de recursos, también recogen información original aplicando encuestas ad hoc. Algunos de éstos, realizados en países industrializados, que intentan medir el peso de factores psicológicos o psicosociales en la causalidad de comportamientos peligrosos en el tránsito, han utilizado también métodos cuantitativos, como la construcción y aplicación de escalas, donde las alternativas de respuesta están prefiguradas por el investigador (GELDSTEIN; BERTONCELLO, 2006, p. 11-16).

La cuestión de la percepción del riesgo también ha sido abordada mayoritariamente desde esta perspectiva. Y, como ha sido señalado en la extensa revisión crítica de Mary Douglas (1996), cuando las ciencias sociales analizan la cuestión de la percepción del riesgo tienden a aplicar teorías y marcos analíticos originados en el cálculo riesgo-beneficio o costo-beneficio, suponiendo que los seres humanos siempre efectúan elecciones racionales de este tipo - donde la "racionalidad" es definida externamente por los expertos o investigadores, quienes suelen dejar de lado las diferencias culturales y las diferentes evaluaciones subjetivas del riesgo derivadas de dichas diferencias, cuyo abordaje necesita de estrategias cualitativas o, mejor aún, de la triangulación de estrategias cuantitativas y 
cualitativas que integren los conocimientos de diversas disciplinas- (DOUGLAS, 1996). Los estudios de este tipo parecen ser muy escasos a nivel internacional. ${ }^{4}$

En América Latina también prevalecen las investigaciones cuantitativas, aunque se encuentran algunos trabajos de tipo conceptual y unos pocos que aplicaron métodos cualitativos (por ejemplo, O’DONNELL, 1989; FRAIMAN; ROSSAL, 2005; MOYANO DÍAZ et al., 1999; WRIGHT et al., 2007) y también propuestas de políticas de promoción de la seguridad vial y, en mucha menor medida, descripciones de experiencias de intervención exitosas (por ejemplo, MOCKUS, 2001, 2002a, 2002b) Los estudios cualitativos detectados son francamente escasos. Esta situación se repite en Argentina, donde ya es importante la producción de investigación sobre el tema desde una perspectiva cuantitativa, pero todavía se encuentran escasísimos estudios publicados que empleen perspectivas de investigación cualitativa (por ejemplo, BOSQUE, 2006; DE HOYOS, 2006; GELDSTEIN, 2006; PELTZER; 2006; REY, 2006).

En este sentido, la forma en que operan las estructuras de género en la subjetividad y las prácticas de los agentes, si bien es una dimensión aún escasamente estudiada en relación al tránsito urbano, podría contarse entre las más relevantes y fructíferas para comprender las prácticas de riesgo y ciertos hechos de violencia vial y, en esa medida, contribuir a su prevención. Para intentar tal abordaje, resultan de gran valor algunas herramientas analíticas propuestas por Pierre Bourdieu. Entre ellas, aquí nos apoyamos en el concepto de habitus, para superar la separación entre conciencia y práctica y contribuir al análisis de la dialéctica entre los sujetos y las condiciones sociales en las cuales se insertan (BOURDIEU, 1991; 2007).5 Las sociedades y sus instituciones producen y reproducen un conjunto de categorías de percepción, motivaciones, intereses, prácticas impensadas, que son incorporadas en los cuerpos y las conciencias de los agentes como una segunda naturaleza. La participación de los agentes en el juego en un campo y en un espacio social depende de la incorporación (más allá de la conciencia y la voluntad) de una doxa, conjunto de reglas prácticas, de axiomas que no se cuestionan y que, como las categorías de percepción y valoración del resto de los capitales que están en juego en cada campo, son generadas por el capital simbólico. Es así que "la aceptación dóxica del mundo, que resulta del acuerdo inmediato de las estructuras objetivas con las estructuras cognoscitivas" resultaría clave para una explicación realista de la dominación (BOURDIEU; 
WACQUANT, 1995, p. 120) y para la comprensión de la participación de los agentes (y especialmente, de los dominados) en la reproducción del campo y de sus formas de dominación (BOURDIEU, 2000; 2007).

En su intento de llenar un vacío en la teorización de la violencia interpersonal (y de género) en las ciencias sociales, Henrietta Moore (1994) plantea la necesidad de contar con una teoría del sujeto. En esta dirección son sumamente útiles sus señalamientos acerca del papel de las diferencias dicotómicas en la constitución de la subjetividad (ver también BOURDIEU, 2000; LAMAS, 1999) Según esta autora, el poder (performativo) de los discursos acerca del género reside, entre otras cosas, en que ellos producen mujeres y hombres como personas definidas por la diferencia - aunque los comportamientos, las cualidades, los atributos y las imágenes de sí misma/o de las personas reales no guardan una relación estrecha con las construcciones sociales de lo masculino y lo femenino (MOORE, 1994, p. 138-139). ${ }^{6}$ En este sentido, sostiene la coexistencia de múltiples identidades subjetivas y discursos de género, tanto a nivel de la sociedad como de los individuos, que pueden asumir (y de hecho, lo hacen) diferentes posiciones de sujeto en sus prácticas discursivas. Ello permite diferenciar entre el discurso de género dominante y los discursos sub-dominantes acerca de la masculinidad y la feminidad. E implica reconocer la existencia de diversidad y la posibilidad de resistencia a los modelos hegemónicos y los comportamientos prescriptos para las categorías opuestas de mujer y de hombre: en esa medida, la reproducción social del genero podría ser también re-producción, cambio (HOLLWAY, 1984).

En el contexto de este trabajo denominamos "posiciones discursivas dominantes" a las que representan, justifican y suscriben, en el campo social del tránsito, el tipo de valores y de prácticas usualmente identificados con la "masculinidad hegemónica" (fuerza, poder, agresividad, competitividad, toma de riesgo, valentía, belicosidad, decisión, eficiencia, violencia, voluntad de dominio, entre otros) y utilizamos el término de "posiciones discursivas sub-dominantes" para aquellas que, sin ser necesariamente minoritarias en términos de frecuencia, expresan identidades, valores y comportamientos opuestos, alternativos o de resistencia a las prácticas y los discursos dominantes. De la misma manera en que descubrimos los valores de la masculinidad dominante tanto en discursos de hombres como de mujeres, los discursos sub-dominantes, alternativos, fueron expresados, con motivaciones dispares, por mujeres de diferentes generaciones y 
también por hombres; lo que los identifica, lo que tienen en común los emisores

de los discursos sub-dominantes, es tanto su pertenencia a categorías sociales (mujeres, viejos) consideradas "débiles", "feminizadas" o desempoderadas y, en esa medida, estigmatizadas de acuerdo a los parámetros del discurso masculino dominante, como su resistencia discursiva a dicha estigmatización (MOORE, 1994, p. 146; CONNELL, 1987, citado por MOORE, 1994).

Este artículo presenta parte de los resultados cualitativos de dos Estudios Colaborativos Multicéntricos sobre aspectos socioculturales y demográficos de los accidentes de tránsito en Argentina; más específicamente, los obtenidos en entrevistas en profundidad a 92 automovilistas en la Ciudad Autónoma de Buenos Aires (CABA). ${ }^{7}$ Aquí nos centramos en nuestro análisis en torno a dos de las categorías centrales identificadas - a) violencia y b) los otros en el tránsito - , haciendo hincapié en sus vinculaciones con la dimensión de género. A partir de un trabajo de comparación constante, dialogando con las citadas herramientas conceptuales de la teoría social contemporánea, identificamos dos grandes tipos de posiciones discursivas - dominantes y sub-dominantes que atraviesan los discursos de los distintos grupos de entrevistados (según sexo y generación) A continuación describimos algunas de las categorías emergentes, agrupándolas en torno a cada uno de dichos grandes tipos de posiciones discursivas. En la tercera sección, a modo de cierre, proponemos una articulación entre dichas categorías, reflexionando acerca de las posibles vinculaciones entre la dominación masculina, los estilos de conducción y la reproducción de las prácticas de riesgo en el campo del tránsito urbano.

\section{Posiciones discursivas dominantes ${ }^{8}$}

\section{La calle, ¿es cosa de hombres?: la violencia en el tránsito}

El tránsito vehicular de Buenos Aires es percibido e interpretado por la mayoría de los conductores de automóviles particulares como un campo social caótico y violento, características atribuidas a las condiciones culturales y sociales que propician prácticas trasgresoras, violentas, egoistas, faltas de respeto o de civismo. Mujeres y varones tienen percepciones diferenciadas en torno a la violencia interpersonal en el tránsito. Como se ilustra en los fragmentos de entrevistas citados en el recuadro 1, las conductoras, sin distinción de edad refieren múltiples situaciones de violencia de 
las que fueron víctimas y que suelen interpretar como discriminación de género. Los (más escasos) relatos de los hombres como receptores de violencia refieren a confrontaciones de poder masculino real o simbólico (mayor tamaño del vehículo, mayor fuerza física, mayor número y/o juventud del oponente). Ellos raramente reconocen ejercer violencia interpersonal contra las mujeres; cuando abordan la cuestión tienden a hacerlo con un discurso sobre la discriminación de la que los hombres en general - o los otros hombres- las hacen objeto, asumiendo en lo personal una posición subjetiva "políticamente correcta", es decir contraria al machismo y a la estigmatización de las mujeres que conducen.

\section{Recuadro 1}

[...] muchas veces me gritaron cosas hípermachistas [...] mucha prepotencia, mucho prejuicio [...] pensás "no puede ser que me estás puteando así porque pasé yo y no te dejé pasar" [...] está descargando su presión del día en mí porque soy mujer y sabe que no me voy a bajar para pelear (Juana, 20).

Sí, trato de ser prudente, pero igual los hombres suelen ser arrolladores [...] Te descalifican por cualquier cosa [...] y el de atrás me toca bocina [...] No lo puedo pasar [al de adelante] y me miran como si... [sugiere que la miran así porque es mujer] (Micaela, 24).

La 4x4 [...] me siguió y me empezó a tirar el auto [...] cuando lo terminé pasando y me empezó a tocar bocina [...] vio que era una mina y no le gustó y me vino a buscar de nuevo (Roxana, 28).

[...] sí, [me pasó] que me encerraran, le hice luces a un taxista que venía por una avenida, por la izquierda, por el carril rápido, le hice seña para que me dejara pasar, para que se corriera, me deja pasar, y en el momento que me deja pasar me vuelve a encerrar y me hace irme de la doble línea amarilla del carril contrario. Es decir, si venía uno de frente choco o choco contra el taxi que me encierra. Y más cuando sos mujer (Elisa, 30).

[...] el nivel de agresividad que hay en los hombres, que en otro momento, uno por ahí hacía una mala maniobra y el tipo te puteaba, y ahora son capaces de cerrarte con el auto y bajar, a mí me pasó, y yo subir la ventanilla porque "este tipo me va a dar una trompada" [...] me ha pasado, de cruzarme el auto por prepotencia, de decir: "No pasas" (Susana, 49).

[violencia] ¡En la agresividad! O sea, te tratan como si fueras una estúpida, una idiota. Te dicen “¡Andá a lavar los platos!” [...] es lo mínimo que te dicen [...] Te desvalorizan continuamente (Serena, 50).

[...] en una época me indignaba o insultaba [...] pero ahora no, nada... me encojo de hombros y sigo... [...] (Bernardo, 73).

El motoquero, pasó uno y me tocó el espejo y le digo: “¿Qué hacés, sos b...?”, "Qué te pasa, viejo p...?”. Y ahí nomás ya tenía cinco o seis sobre la Avenida Córdoba, porque es una patota, se ayuda. Me salvé de que me patearan el auto. Eso es violencia (Ramiro, 60). 
Asimismo, como se observa en los fragmentos de entrevistas citados en el recuadro 2, si bien hombres y mujeres opinan que es mejor no establecer contactos que podrían derivar en más violencia, suelen admitir que reaccionan, al menos verbalmente, cuando se sienten agredidos. Los varones de edades medias y maduras dicen conducirse con cierta precaución a la hora de las discusiones, mientras los jóvenes parecen más compelidos a sostener un estereotipo masculino competitivo y belicoso, reaccionando con violencia ante situaciones de agresión, contacto visual o verbal y/o de los autos.

\section{Recuadro 2}

Más de joven, por ahí reaccionaba, me enojaba, gritaba, ahora no. Si alguien me increpa por alguna supuesta maniobra [...] le pido disculpas, y ya está. Trato de no hacerme mala sangre (Eriberto, 73).

No, el tipo me estaba mirando, y le digo "qué hacés" y me mira como diciendo "no me hinches las bolas". No, lo quería matar, pero [...] lo puteé y me fui. El problema es que un día uno reacciona y ahí no sé qué va a pasar. Yo [...] eso de bajarse con el matafuego, bajarte con un fierro, no [...] (Pedro, 40).

Sí, yo soy de pasar tranquilo, pero cuando me faltan el respeto me transformo, no me gusta que me falten el respeto. Me he bajado, más de dos piñas nunca. Sí, bajar a discutir, porque encima te encierran y creen que tienen razón (Guillermo, 22).

Las mujeres reconocen que reaccionan con vehemencia ante ciertas agresiones. Pero, a diferencia de los hombres, parecen reprimir la exteriorización de sus respuestas. Como se ilustra en el recuadro 3, si bien reconocen experimentar sentimientos violentos, no llegan a situaciones de confrontación directa, que se inhiben de llevar a la práctica por temor a la reacción y superioridad física de sus ocasionales oponentes masculinos, limitándose a "expresiones gestuales o verbales dentro del vehículo". Si el otro es un hombre, la violencia verbal es actuada hacia dentro: pueden verbalizar insultos pero sin que el otro los escuche. Pero sus actitudes son más abiertas si el conflicto es con otra mujer. La posibilidad de ejercer violencia física es vivida por ellas como fantasía: la de tener un poder masculino que repararía simbólicamente el sentimiento de impotencia. 
O te tocan bocina y hacen luces todo el tiempo, no es necesario, si el semáforo cambia a verde yo lo veo $[. .$.$] a mí me pone de muy mal humor, me sacan ese instinto asesino que tengo guardado$ (Micaela, 24).

Pero a veces te da bronca. A veces alguna mala palabra lanzás. "Por qué no te corrés... no se qué", y por ahí lo decías adentro del auto [...] (Marta, 36).

[...] a veces yo digo "si yo tuviera un camión se lo arrimo a la parte de atrás para asustarlo" [...] no es que mi intención sería tener un camión para tocarlo al otro, pero sino intimidar [...] para que no me encierren por la derecha como a veces hacen (Andrea, 36).

[...] Esta impotencia de saber que vos tenés razón [...] al hombre le toco bocina [...] a la mujer bajo la ventanilla y la puteo [...] hay cosas que a mi me enervan y si fuera hombre creo que llegaría a la violencia [...] Esto de que un tipo te encierre, creo que si fuera hombre hasta que no le termino de tocar bocina no paro y por ahí el otro es más loco que yo [...] A veces me pregunto qué sería de mí si fuera hombre, manejando (Elisa, 30).

[...] si yo pudiera me bajaría y les pegaría [...] no tienen derecho a andar pasándote, haciendo cosas totalmente extrañas, poniendo en riesgo a un montón de gente, porque ellos van como jugando, como si estuvieran en un juego. (Nadia, 45).

Bocinazo y puteo para mí [gritarle al otro] ¡No, eso no! (Morena, 32).

\section{La ley de la calle: la dominación masculina en el tránsito}

Las conductas prepotentes e imprevistas de otros están entre las situaciones que más discordia, disconformidad, o violencia parecen generar en los automovilistas, pues impedirían distenderse en la confianza de una norma compartida. ${ }^{9}$ Sin embargo, tal como se observa en los fragmentos de entrevistas citados en el recuadro 4, la significación extendida en los discursos de mujeres de todas las edades de que manejar bien es manejar con destreza frente a lo imprevisto y adaptándose con rapidez, habilidad y eficiencia a las condiciones reales se constituye en justificación pragmática de la trasgresión, que es significada como una forma de supervivencia incorporada como habitus.

Si las identidades de género se construyen y sostienen a través de las oposiciones dicotómicas (MOORE, 1994; BOURDIEU, 2000; LAMAS, 1999) y la dominación masculina se expresa y afirma mediante discursos de poder que estigmatizan a las mujeres, o que tienden a feminizar a los débiles y los dominados (MOORE, 1994), ello se descubre en las prácticas discursivas en torno a la interacción en el tránsito, que también refieren a otras diferencias sociales (clase, poder, generación), incluyendo estereotipos dicotómicos de los tipos de vehículos 
y las conductas de quienes los manejan. ${ }^{10}$ Por una parte, los autos nuevos y las $4 \times 4$, que pueden viajar a más velocidad - conducidos con prepotencia por propietarios seguros de sus prerrogativas de estatus - y los colectivos, cuyos conductores son capaces de ejercer violencia (incluso contra otros hombres) en razón del tamaño de sus vehículos. Por la otra, los autos viejos y mal mantenidos que, piloteados por viejos vacilantes y sin reflejos, circulan a menor velocidad que la exigida por la calle. Como se ilustra en los fragmentos de entrevistas citados en el recuadro 5 , similares prácticas valoradas negativamente se atribuyen a las mujeres - en especial a las mayores.

\section{Recuadro 4}

Manejar bien para mí es conocer las reglas de juego reales en la calle [...] no siempre son las que recomienda la ley de tránsito [...] poder cambiar de carril fácilmente, eh... [...] ciertos detalles y ciertos códigos de la calle que ... hacen que el tránsito sea un poco más fluido [...] quizá no es lo más prolijo, este... pero... [...] por lo saturado que está el tránsito [...] Y hay una cosa muy tácita ya [...] aunque el de la derecha es el que tiene el derecho a pasar, hay como una cosa de timing de quién llegó un segundo antes o si hay una cuneta, porque en general el que tiene una cuneta para y el que no la tiene sigue y, digamos, eso no está especificado en la ley. $\mathrm{Y}$ eso todos lo saben sin haberlo aprendido... como que lo va mamando de la calle $[. .$.$] a veces la gente [. .$.$] este... no se$ anima a hacer ciertas maniobras que quizá no son las mejores pero [son] necesarias para evitar un atascamiento peor [...] (Roxana, 28).

[...] Y..., poder reaccionar adecuadamente a los imprevistos que surjan [...] poder frenar a tiempo o maniobrar correctamente para no provocar daños mayores. [...] en Estados Unidos [...] la gente está tan atada a las reglas [que] no saben manejar los imprevistos [...] En cambio acá, como uno está acostumbrado a la improvisación constante y a los imprevistos, por ahí no hay tantos accidentes por pavadas. [Manejar mal es] estar demasiado estructurado a seguir las reglas, esto te impide manejar los imprevistos (Marta, 36).

\section{Recuadiro 5}

Y aparte, uno se da cuenta - capaz - que es mujer... dice "ésta es mujer" y no le vio la cara... ¡y sí, es mujer! Y la mayoría de los errores, capaz que son de mujeres (Esteban, 23).

La mujer maneja mucho mejor que el hombre en lo que respecta al respeto de las normas [...] Lo que tiene que [...] en el manejo del auto es mucho más torpe, pero mucho más torpe. Una mujer maneja con miedo, maneja asustada, maneja muy dura. [...] Ahora, si todo el mundo manejase así no afectaría en nada. El tema es que por la forma en que maneja todo el mundo te termina afectando. La mujer [...] el semáforo se pone amarillo y ya pisa el freno. El hombre [...] pisa el acelerador para poder pasar. Para el hombre es como que el amarillo es "dale que paso", así pasás. Y para la mujer, el amarillo es "guarda que viene el rojo" y frenás. Y una mujer y un hombre atrás, la mujer frenó... [risas] (Pedro, 40). 
Aunque el respeto a la norma jurídica constituiría una situación ideal, las condiciones reales del campo lo tornarían ineficiente y peligroso y las prácticas respetuosas de unos pocos se significan entonces como un excesivo apego a las normas y se interpretan como manejar mal. La percepción de que es preciso entender los códigos de la calle y adquirir los hábitos de manejo que permitan desempeñarse conforme a ellos, aparece así como la aceptación dóxica de una necesidad del campo, constituyéndose a la vez en un mecanismo para su reproducción (BOURDIEU; WACQUANT, 1995). Dichos códigos de la calle son en realidad códigos masculinos, asociados a estilos de conducción caracterizados por el riesgo, la competencia, el pragmatismo, la seguridad (en sí mismo/a) y la habilidad o la destreza, coincidentes con los valores de la masculinidad hegemónica característicos del capitalismo occidental (MOORE, 1994). El discurso dominante atribuye este estilo de conducción a los hombres quienes, en virtud de disposiciones naturales serían quienes manejan bien. Esta identidad del buen conductor se construye en contraposición con un estereotipo femenino, connotado de forma (aparentemente) positiva en relación a lo cauteloso o negativa, en relación al temor, la inseguridad y la distracción. Como se observa en el recuadro 6, las críticas se encontraron frecuentemente en los discursos de varones jóvenes, quienes definen a las mujeres como personas con miedo, inseguras, carentes de habilidad.

Asimismo, como se ilustra en los fragmentos de entrevistas citados en el recuadro 7, las mujeres maduras suscriben el discurso dominante y parecen confirmar el estigma que pesa sobre ellas, pues si bien definen a los hombres como imprudentes o más arriesgados, naturalizan una supuesta mayor aptitud masculina en la conducción, mientras se definen a sí mismas - en tanto categoría de género- como prudentes pero despistadas o distraídas. 
[...] para mí, ciertas mujeres, están muy pendientes de, de..., eh... en el momento de hacer una maniobra, ¿no?, en no mirar el espejo, sino en, en... realizar ciertos movimientos... [...] Y tener la mente en el embrague, en el acelerador, o en los cambios, y no en [...] la macana que se puede llegar a mandar el de adelante [...] yo ese defecto lo veo más en las mujeres... [...] Como que el hombre intuye más lo que puede llegar a hacer el vehículo que tiene al lado (Néstor, 22).

Sí, distraída. Como que están pensando en "qué voy a cocinar hoy a la noche...; cómo voy a encontrar a mi marido; qué le habrá pasado al nene en el colegio...” [...] Y a las más viejas... las veo atentas... demasiado atentas (Carlos, 25).

[...] son bastante distraídas para manejar, no prestan demasiada atención [...] te das cuenta que la mina se va arreglando el flequillo y ni está mirando la calle, [...] o viene discutiendo con los nenes [...] las mujeres más jóvenes no son tan malas manejando, es más yo creo que son hasta mejores que los hombres manejando, las mujeres de cierta edad treinta y cinco, cuarenta años para arriba es bastante desprolija para manejar (Jeremías, 35).

No es por ser machista... pero... yo veo en mujeres quizás mucho miedo..., mucha tensión cuando maneja. Quizás por una... eh... exigencia máxima de responsabilidad... de que eso puede llevar al miedo también... [...] tienen mucho miedo de manejar. (Damián, 18).

[...] es más responsable [...] pero [...] pienso yo que es más cagona, más miedosa, temerosa. En la esquina frena porque puede venir alguien, y vos ves que está en el desierto (Santiago, 39).

\section{Recuadro 7}

Es un poquito más calma la mujer para manejar. Inclusive hay mujeres que son un poco torpes, también. (Serena, 50).

[...] las mujeres de por sí, en todos los órdenes de la vida me parece que somos más prudentes. El hombre es más temerario, pero por una cuestión de naturaleza me parece, que responde más a algo natural. Entonces se nota en el manejo. Quizás las mujeres somos despistadas manejando, entonces, por ahí no nos dimos cuenta de alguna cosa [...] los hombres no, los hombres no son despistados, son muy vivos para manejar, pero son imprudentes (Susana, 49).

No quiero ser ni machista ni antimachista, pero creo que los hombres se sienten más seguros frente al volante, [...] en un hábitat más natural que para la mujer. La mujer, me parece que la mayoría, como yo, es un artefacto que utilizan [...] las señales de tránsito no las conocen tanto como los hombres... [...] Por ejemplo, él aprendió a manejar mucho después que yo, aprendió después de que nos casamos [...]... y maneja tanto mejor que yo, y yo ya tenía unos 20 o 15 años de manejo cuando él empezó [...] La mujer va más así, uh, muy ansiosas cuando manejan. No sé por qué. Será porque es socialmente... a las mujeres las cargan hay tantos chistes [...] Pero es verdad que lavo mejor los platos que lo que manejo [...] y a veces hago más cosas para que me lo digan, así jodo un poco (risas) (Sabrina, 60). 
Estos discursos femeninos recuerdan el papel activo que Bourdieu (2000) asigna a los dominados en la reproducción de la relación de dominación, ${ }^{11}$ incluso en su referencia a la profecía autocumplida:

Cuando los dominados aplican a lo que les domina unos esquemas que son el producto de la dominación, o, en otras palabras, cuando sus pensamientos y sus percepciones están estructurados de acuerdo con las propias estructuras de la relación de dominación que se les ha impuesto, sus actos de conocimiento son, inevitablemente, unos actos de reconocimiento, de sumisión ( BOURDIEU, 2000, p. 26).

\section{Posiciones discursivas sub-dominantes}

\section{Las jóvenes manejan bien, pero las mayores son demasiado precavidas}

Los hombres mayores hacen una valoración diferente del manejo de las mujeres y en general tienden a relativizar las diferencias en función del género, resaltando prácticas viales como el cumplimiento de normas y la prudencia (virtudes femeninas) frente el riesgo o la imprudencia, que caracterizarían el estilo masculino (ver recuadro 8). Pero entre estos varones aparecen discursos contradictorios, en los que las mujeres (jóvenes) que manejan bien son descriptas con miradas masculinas acerca de lo que es manejar bien, a la par que sugieren que el temor, que caracterizaría a las conductoras maduras, estaría asociado a la lucha por la inclusión y el reconocimiento que ellas libran día a día en el campo masculino del tránsito.

\section{Recuadro 8}

[...] probablemente las más jóvenes sean más hábiles... cuidadosas deben ser [...] las más grandes también... pero a mayor edad mas lentitud para reaccionar [...] a veces, por cuidarse mucho demoran un poco el transito... (Ovidio, 56).

Hay un preconcepto sobre la mujer... hay mujeres que manejan mejor que un hombre. [...] mi hija Paula, maneja perfectamente bien. Maneja mucho mejor que muchos hombres. La edad influye [por] la capacidad, la rapidez [...] (Ramiro, 60).

La mujer es mucho más lenta [...] pero pienso yo que es por precaución. Tiene miedo de meter la pata porque le vamos a caer con todo: "Andá a lavar los platos", "Qué hacés ahí arriba"... [...] a veces $[\ldots]$ hay un cierto cuidado y tiene que ser una mujer [...] la mujer es más precavida, [...] creo que lo hacen por una cuestión de no meter la gamba [...] nadie quiere chocar. Y las mujeres menos que menos porque... (Facundo, 76). 
Las mujeres manejan agarraditas y atentas. Yo, en cambio,

\section{manejo como hombre}

Como se ilustra en el recuadro 9, en los discursos de las conductoras jóvenes hay una alta valoración del pragmatismo en el manejo, que reconocen en los hombres y con el cual desean identificarse, mediante su afirmación personal como buenas conductoras, y la diferenciación respecto de las prácticas de otras mujeres que, en sus representaciones, justificarían el estigma de género que pesa sobre todas ellas.

\section{Recuadiro 9}

[...] la mujer va pegada al volante [...] como que, por ahí es más miedosa, entonces eso genera que vaya con más cautela manejando, entonces le hace ser mas lenta, menos reflejo, no sé esto del miedo, que lo proyectan en el manejar... (Elisa, 30).

[...] Me da la sensación, no todas y no creo formar parte del grupo, sí me pasa que me descalifican por eso. Muchas veces, lo veo mucho en mi mamá también, van por cualquier lado [...] “ipor qué?, hacélo distinto, defendénos" (Micaela, 24).

[...] no tengo muchos problemas yo. Yo manejo como hombre, mi mamá también, que es la que me enseñó [significa] Más seguridad, más confianza... [...] yo manejo con una mano en el volante, nunca agarro con las dos manos, [...] sólo lo agarro cuando voy en la ruta a mucha velocidad [...] Pero sino como voy siempre metiendo cambios, yo voy con una mano y... qué se yo... las mujeres van con las dos manos agarraditas y atentas... [...] Aprendí con otra mina que conduce como varón. Y [...]... es una cuestión de personalidad. Yo soy bastante segura en muchas cosas, soy extrovertida... digo, no sólo con el manejo... eso ayuda [...] y también que fui enseñada con este estilo [...] tuve referentes masculinos, entre mi papá y mi mamá que maneja como un hombre [...] (Roxana, 28).

Siguiendo las conceptualizaciones de BOURDIEU (2000), en esta incorporación de los parámetros androcéntricos en las valoraciones en torno a los estilos de conducción en las mentes y los cuerpos de los agentes dominados, se manifiesta la eficacia de la violencia simbólica masculina en el campo del tránsito:

Los dominados aplican a las relaciones de dominación unas categorías construidas desde el punto de vista de los dominadores, haciéndolas aparecer de ese modo como naturales. Eso puede llevar a una especie de autodepredación, o sea de autodenigración sistemáticas, especialmente visible [...] en su adhesión a una imagen desvalorizada de la mujer (BOURDIEU, 2000, p. 50-51).

\section{Agarraditas y atentas... ¿o la ética del cuidado?}

Como se ha visto, el discurso masculino dominante tiende a estigmatizar a las mujeres - en especial a las mujeres maduras- por su inseguridad, lentitud y exceso de cautela en el manejo, presuntamente debidos a sentimientos de temor; un 
temor disfuncional que tendría, entonces, un efecto paralizante. En este sentido, la palabra miedo (a manejar, al tránsito) fue una de las más utilizadas para calificar el estilo femenino de conducción. Las consideraciones de Douglas (1996) en torno al concepto de inmunidad subjetiva nos permiten pensar la intensidad con la que es percibido el riesgo en el tránsito en términos de un cierto continuum, cuyos valores extremos serían, por un lado, una suerte de temor paralizante y, por el otro, la negación completa del riesgo. En palabras de Douglas (1996, p. 98),

Los riesgos reclaman a gritos la atención; probables peligros se dan cita de todos los lados, en cada bocanada y a cada paso. El agente racional que prestara atención a todos ellos estaría paralizado [...]

Sin embargo, como puntualiza esta autora, los agentes desarrollan mecanismos - el sentimiento de inmunidad subjetiva, el sentido de estar en control- que les permiten desempeñarse en contextos peligrosos:

Los resultados mejor establecidos de la investigación del riesgo muestran que los individuos tienen un sentido fuerte, pero injustificado, de inmunidad subjetiva. En actividades muy familiares existe la tendencia a minimizar la probabilidad de malos resultados. En apariencia, se subestiman aquellos riesgos que se consideran controlados. [...]Y se subestiman también los riesgos que conllevan los acontecimientos que se dan rara vez [...]

Para una especie bien adaptada para sobrevivir, hacer caso omiso de los acontecimientos de baja frecuencia parece una estrategia altamente razonable. [...] Desde el punto de vista de la supervivencia de la especie, el sentido de inmunidad subjetiva es también adaptativo si permite que los seres humanos se mantengan serenos en medio de los peligros, que osen experimentar, y que no se desestabilicen ante la evidencia de los fracasos (DOUGLAS, 1996, p.57-58)

Douglas (1996, citando a JONES; DAVIS 1961 y a ROTTER 1966), señala la importancia del sentido de un lugar de control para dar respuesta a las situaciones de estrés, afirmando que la expectativa de ejercer el control reduciría la experiencia de estrés. Para afrontarlo en el tránsito, algunos conductores extremarán las acciones destinadas al control de la propia agencia, mientras otros intentarán controlar los factores externos, pudiendo descuidar el necesario monitoreo de sí mismo al confiar demasiado en la propia pericia para conducir, en la potencia de su máquina, etc..Otros, finalmente, aplicarán un sentido de inmunidad subjetiva, negando, minimizando o suspendiendo momentáneamente su percepción de riesgos, como mecanismo adaptativo para poder desempeñarse en un contexto adverso. La literatura especializada y nuestros propios análisis interpretativos de los discursos de los entrevistados sugieren que el sentido de control personal 
de la situación y la sensación de inmunidad subjetiva, en parte necesarios para

desempeñarse en un medio difícil sin quedar paralizado por el miedo o el estrés, pueden encontrarse exacerbados por la sobreexigencia que impone el entorno o por la omnipotencia o compulsividad de algunos individuos $y$, en esa medida, propiciar actitudes y comportamientos que impliquen un incremento del riesgo.

Las reflexiones que anteceden y los motivos que algunas mujeres maduras exponen para explicar sus prácticas de conducción nos permiten reinterpretarlas en términos diferentes de aquellos del discurso dominante. En efecto, otro discurso femenino sub-dominante se descubre en la percepción del riesgo de ciertas mujeres maduras cuando éstas dan cuenta de sus motivos para conducir como lo hacen (ver recuadro 10). Lo hemos caracterizado como el control centrado en sí mismo. Esta disposición sugiere la capacidad de anticipar las consecuencias de la acción para sí misma y para otros y el reconocimiento de la potencialidad de daño de la propia agencia; se expresa mediante un discurso moral, que predica la necesidad de automonitoreo, en razón del respeto a la vida y la integridad de las personas; la propia agencia cobra así sentido como control responsable, sobre todo, de sí mismo.

\section{Recuadiro 10}

[...] es como saber manejar un arma... porque vos en un imprevisto podés causar un accidente, aunque no sea por tu voluntad, te puede pasar. Una falla mecánica imprevista, y el auto se sube arriba de la vereda, y vos estás causando un daño, aunque vos no lo hayas querido, aunque fuera imprevisible [...] porque cualquier cosa que yo haga que no se deba hacer, es peligrosa para mí en principio y puede ser peligrosa para terceros... (Nona, 50)

[...] cuando subís a un auto tenés que pensar que puede pasarte cualquier cosa [...]..yo no diría miedo, es como una prevención [...] Tengo que manejar con la mente lúcida por mí, por la gente que llevo y por la gente de afuera, los peatones. Y el otro que maneja. [...] por las personas en sí. Sean nuestra familia, yo, o el que está afuera. Es decir, yo tengo que respetar al que está afuera [...] (Lila, 53)

Bueno, están los ciclomotores que en las esquinas yo trato siempre de mirar, que aunque la flecha diga que el tránsito va para allá - porque ya me pasó un montón de veces que te aparecen en la contramano - ... o doblan sin luces, y... siempre miro hasta para arriba. [...] Cuando paso al lado de una moto, de estas chiquitas, o un ciclista, trato de estar a 3 metros, a 4, porque a mí me da terror que pase algo, tanto del punto de vista legal que significa eso, o que puedas lastimar a otro, ¿no? [...] En general veo mucha imprudencia también en el ciclista, entonces, pensando siempre en la vulnerabilidad, me asusta, siempre trato de guardar distancia [...] Y, [manejar bien es] tener en cuenta al otro, cosa que no siempre... o sea, yo por ejemplo, cuando conduzco, estoy más pendiente del espejo trasero, los laterales, y un 10 por ciento para adelante [...] O sea, para... en ese sentido soy prudente, trato de prever lo imprevisible. (Coco, 46) 
En estos discursos, la imagen del auto como un arma está referida no al poder de la máquina, sino a la agencia de quien lo maneja y nos recuerda el concepto de ética del cuidado como una disposición femenina guiada por el temor de dañar a otros (GILLIGAN, 1996). Esta conciencia de riesgo se opondría tanto al temor paralizante - que el discurso masculino dominante atribuye a las mujeres, en especial a las mujeres maduras que por ese motivo serían lentas y entorpecerían la circulación vehicular- como a una actitud negadora del riesgo que permitiría conducir[se] de manera eficiente en un contexto peligroso (GELDSTEIN, 2006, p. 178-181).

Esta posición discursiva se opone también a las que pregonan el control centrado en la situación (GELDSTEIN, 2006), identificada en los discursos masculinos (de conductores varones y mujeres jóvenes) El control centrado en la situación deriva de la percepción de los otros (conductores y peatones) como factores de riesgo (lo que implicaría poner el riesgo afuera de sí mismo/a y, por lo tanto, la necesidad de control sobre los factores externos a sí mismo) (ver recuadro 10). Con frecuencia este tipo de discurso sobre el riesgo expresa también una preocupación exclusiva por la propia seguridad o la anticipación de las posibles consecuencias de la acción en términos de inconvenientes para sí mismo (si atropellás a alguien/ si matás a otro te metés en un quilombo). De esta manera, tal como interpretamos en un trabajo anterior:

El sentimiento de estar expuesto a sanción y culpa por otros, llevaría a intensificar aun más el monitoreo como intento de control de los factores externos (aun si percibidos como imprevisibles), desdibujando el sentido de la eficiencia del control sobre si mismo (GELDSTEIN, 2006, p. 179).

\section{Reflexiones finales: dominación masculina, tránsito y riesgo}

Y siempre he visto en la dominación masculina, y en la manera como se ha impuesto y soportado, el mejor ejemplo de aquella sumisión paradójica, consecuencia de lo que llamo la violencia simbólica, [...] invisible para sus propias víctimas, que se ejerce esencialmente a través de los caminos puramente simbólicos de la comunicación y del conocimiento o, más exactamente, del desconocimiento, del reconocimiento o, en último término, del sentimiento (BOURDIEU, 2000, p. 12).

Hemos identificado diversas manifestaciones y mecanismos de la dominación masculina en el campo social del tránsito porteño, tanto en sus aspectos más 
crudos y concretos de violencia interpersonal, como en su dimensión simbólica, oculta a la mirada superficial, que produce y reproduce las condiciones para dicha dominación. Los estereotipos estigmatizados de los lentos y temerosos (no viriles) - los viejos y las mujeres - proporcionan el motivo aparente y la justificación de esa violencia, porque ese campo competitivo, que -en sintonía con la competitividad observable en otros ámbitos de la vida social- necesita que todo sea rápido y eficiente, no podría tolerar a los lentos o indecisos y porque la virilidad necesita afirmarse mediante la diferencia con lo femenino o feminizado (carente de poder) Los varones jóvenes, especialmente, parecen expresar lo que sería el estereotipo paradigmático de un discurso masculino dominante. Ellos estigmatizan y discriminan a las mujeres, tal vez por su necesidad de diferenciarse de ellas para constituirse como sujetos (masculinos) del tránsito de pleno derecho, como una forma de afirmar su virilidad en ese campo - de cuya reproducción así participan- y a través de éste, en el mundo masculino adulto.

Pero el fenómeno de la dominación masculina en el tránsito también emergió de los discursos de mujeres conductoras de diversas generaciones. Dichas agentes construyen sus habitus, doxa y experiencias en dicho campo a partir de la dicotomía entre un modelo exitoso de gestión de la conducción asociado a lo masculino pragmatismo, seguridad y eficacia - y un estereotipo negativo de las mujeres conductoras - excesivamente apegadas a las normas, lentas, inseguras e ineficaces - . Este fenómeno, además de contribuir al mantenimiento de la relación de fuerza hegemónica en el campo, aporta a la reproducción de habitus y doxa contrarios al respeto por las normas y por los demás agentes que constituyen el tránsito. Ello ocurre a través de dos mecanismos diferenciados entre mujeres maduras y jóvenes, quienes asumen sendas posiciones subjetivas ante esos dos estereotipos.

Las mujeres maduras que adoptan el discurso dominante suscriben y naturalizan la supuesta superioridad de los hombres al volante, aceptando para sí mismas una posición femenina subordinada; al hacerlo, legitiman la dominación masculina en el tránsito y contribuyen así a su mantenimiento y reproducción.

El discurso sub-dominante de las mujeres jóvenes expresaría la existencia de subjetividades alternativas, de resistencia al discurso dominante que estigmatiza a las mujeres mediante un estereotipo negativo. Pero para ser aceptadas y legitimadas como buenas conductoras, ellas intentan diferenciarse de lo femenino desvalorizado mediante la adopción del discurso y, lo más importante, de las 
prácticas del dominador (como hacen tantas mujeres para escalar posiciones en otros campos sociales de hegemonía masculina: la política, los negocios) y al hacerlo contribuyen, ellas también, a la reafirmación de aquella imagen femenina negativa y a la reproducción del estilo masculino dominante. En este sentido, en la medida en que los varones jóvenes continúen incorporando habitus en torno a la representación dominante de la virilidad y las mujeres jóvenes se sigan inclinando por la adopción de estos mismos patrones masculinos de conducción como una estrategia de adaptación a las condiciones del campo y para eludir el estigma de género, se puede predecir el incremento de la competencia, la violencia y el riesgo en el tránsito ciudadano.

El otro discurso femenino sub-dominante, el que recuerda el concepto de la ética femenina del cuidado, encontrado entre algunas mujeres maduras y - justo es decirlo-, también entre unos pocos hombres afirma, por el contrario, cualidades femeninas positivas, opuestas al estilo masculino individualista y riesgoso. $\mathrm{Y}$ en esa medida sugiere algunos valores culturales positivos a enfatizar en las intervenciones de prevención de AT y, en general, en los ámbitos de formación de los ciudadanos, para que las condiciones del campo social del tránsito se reproduzcan de una manera diferente y más promisoria para la seguridad vial.

Las definiciones de Niklas Luhman (1996) en torno a los conceptos de riesgo y peligro $^{12}$ resultan de utilidad para enriquecer la reflexión sobre las posibles vinculaciones entre el fenómeno de la dominación masculina y los índices de evolución de los accidentes de tránsito en Argentina. Para el sociólogo alemán, los accidentes pueden conceptualizarse como riesgo o como peligro. Si se considera que dichos episodios son provocados por circunstancias externas y que los sujetos que sufren sus consecuencias son víctimas de condiciones que no decidieron, se habla de peligro. Los accidentes de tránsito, según esta posición discursiva, serían sucesos en los que no interviene la voluntad humana, algo imprevisto, azaroso, sin responsables (BOSQUE, 2006) En cambio, si se los define desde la categoría de riesgo, se implica que hay, al menos en parte, responsabilidad humana, erradicándose la idea de que son hechos inevitables y abriéndose, por ende, la posibilidad de la prevención. En términos de Bourdieu (BOURDIEU; WACQUANT, 1995), la hegemonía de una u otra posición discursiva depende de diversas condiciones históricas y de las disputas y relaciones de fuerza en los diversos campos del espacio social, ocupando un lugar estratégico el capital simbólico, político. 
A nuestro entender, a partir de la visibilización de los diversos y múltiples mecanismos mediante los cuales la dominación masculina se incorpora, en las mentes y en los cuerpos de los conductores de automóviles - especialmente los jóvenes - , reproduciendo la doxa y los habitus dominantes en el campo del tránsito - pragmatismo, impugnación de las normas, competencia, violencia , las ciencias sociales pueden contribuir a la desnaturalización de las actuales condiciones del campo y por lo tanto, al pasaje de la definición de los AT como peligros inevitables a su comprensión como riesgos sobre los que es posible intervenir preventivamente. En este sentido es posible imputar, en términos de Max Weber (1993), una afinidad electiva entre las reglas del juego reproducidas por las representaciones androcéntricas del tránsito, presentes tanto en las posiciones discursivas dominantes como sub-dominantes, y los elevados niveles de participación de conductores jóvenes - en su gran mayoría varones, pero crecientemente también mujeres - en los AT producidos en Argentina. ${ }^{13}$

Siguiendo las reflexiones de Bourdieu (2000), si bien la divulgación de los análisis científicos de una forma de dominación puede reforzar simbólicamente dicha dominación, cuando sus verificaciones parecen recuperar o retocar los discursos dominantes, también pueden contribuir a neutralizarlos, al favorecer la reacción de las víctimas:

Sólo una acción política que tome realmente en consideración los efectos de dominación
que se ejercen través de la complicidad objetiva entre las estructuras asimiladas (tanto en
el caso de las mujeres como en el de los hombres) y las estructuras de las grandes institu-
ciones en las que se realiza y se reproduce no sólo el orden masculino, sino también todo
el orden social [...] podrá, sin duda a largo plazo, y amparándose en las contradicciones
inherentes a los diferentes mecanismos o instituciones implicados, contribuir a la extin-
ción progresiva de la dominación masculina (BOURDIEU, 2000, p. 141).

\section{Referencias}

ALTIERI, D. M. Aspectos demográficos de los accidentes de tránsito en Argentina. In: GELDSTEIN, R. (Coord.) Accidentes de tránsito. Oportunidades, recursos y barreras para su ocurrencia, consecuencias, conocimiento y prevención. Informe final de investigación. Buenos Aires: CENEP/Comisión Nacional Salud Investiga, Ministerio de Salud y Ambiente de la Nación. Parte I, 2008. p. 35-44.

BOSQUE, L. Víctimas atendidas en hospitales públicos de la ciudad de Buenos Aires. In: GELDSTEIN, R.; BERTONCELLO, R. (Coord.). Aspectos demográficos y sociales de los accidentes de tránsito en áreas seleccionadas de la Argentina. Buenos Aires: Comisión Nacional de Programas de Investigación Sanitaria, 2006. p. 189-211. 
BOURDIEU, P. La distinción: criterio y bases sociales del gusto. Madrid: Taurus, 1991.

BOURDIEU, P. La dominación masculina. Barcelona: Anagrama, 2000.

BOURDIEU, P. El sentido práctico. Buenos Aires: Siglo XXI, 2007.

BOURDIEU, P.; WACQUANT, L. Respuestas. Por una antropología reflexiva. México: Grijalbo, 1995.

DE HOYOS, G. Motociclistas jóvenes en la ciudad de Neuquén. In: GELDSTEIN, R.; BERTONCELLO, R. (Coord.). Aspectos demográficos y sociales de los accidentes de tránsito en áreas seleccionadas de la Argentina. Buenos Aires: Comisión Nacional de Programas de Investigación Sanitaria (CONAPRIS). Ministerio de Salud y Ambiente de la Nación, 2006. p. 87-115.

DOUGLAS, M. La aceptabilidad del riesgo según las ciencias sociales. Paidós: Barcelona, 1996. FRAIMAN, R.; ROSSAL, M. De las micro-escenas a los macro-dramas. Observación participante y 'realidad' social. Trabajo de investigación del proyecto Cultura y violencia en el tránsito en Montevideo. In: VI reunión de Antropología del MERCOSUR, Montevideo, 2005.

GELDSTEIN, R. Automovilistas adultos y peatones adultos mayores en la ciudad de Buenos Aires. In: GELDSTEIN, R.; BERTONCELLO, R. (Coord.). Aspectos demográficos $y$ sociales de los accidentes de tránsito en áreas seleccionadas de la Argentina. Buenos Aires: Comisión Nacional de Programas de Investigación Sanitaria (CONAPRIS), Ministerio de Salud y Ambiente de la Nación, 2006. p. 151-187.

GELDSTEIN, R. (Coord.) Accidentes de tránsito. Oportunidades, recursos y barreras para su ocurrencia, consecuencias, conocimiento y prevención. Informe final de investigación. Buenos Aires: Ministerio de Salud y Ambiente de la Nación, 2008.

GELDSTEIN, R.; BERTONCELLO, R. (Coord.) Aspectos demográficos y sociales de los accidentes de tránsito en áreas seleccionadas de la Argentina. Buenos Aires: Ministerio de Salud y Ambiente de la Nación, 2006. Disponible en: http://www.saludinvestiga.org.ar/ pdf/libros/2006/Geldstein-Bertoncello.pdf Acceso el: 13 jun 2011.

GILLIGAN, C. In a different voice. Psychological theory and women's development. Cambridge, Massachusetts y Londres: Harvard University Press, 1996 [1982].

GLASER, B.; STRAUSS, A. The discovery of grounded theory, New York: Aldine Publishing Company, 1967.

HOLLWAY, W. Gender difference and the production of subjectivity. In: HENRIQUES, J., HOLLWAY, W.; VENN, C. Changing the subject: Psychology, Social Regulation and Subjectivity. Londres: Methuen, 1984. p. 227-263.

LACASTA, L. Los costos en vidas, salud y atención de los accidentes de tránsito en la Argentina. In: GELDSTEIN, R.; BERTONCELLO, R. (Coord.) Aspectos demográficos $y$ sociales de los accidentes de tránsito en áreas seleccionadas de la Argentina. Buenos Aires: Ministerio de Salud y Ambiente de la Nación, 2006. p. 23-32. 
LAMAS, M. Género: los conflictos y desafíos del nuevo paradigma. In: PORTUGAL, A. M.; TORRES, C. (eds.). El siglo de las mujeres. Santiago de Chile: Ediciones de las mujeres, n. 28, p. 87-99, 1999.

LUHMANN, N. Sociología del riesgo. México D.F.: Universidad Iberoamericana, 1996.

MAXWELL, J. A. Qualitative research design. An interactive approach, London: Sage, 1996.

MENDES DIZ, A.M. Los jóvenes y las normas. Crónica de un desencuentro anunciado: el caso de los accidentes de tránsito. In: KORNBLIT, A. L. (Coord.). Juventudy vida cotidiana. Buenos Aires: Biblos, 2007. p. 103-118.

MOCKUS, A. Cultura ciudadana, programa contra la violencia en Santa Fe de Bogotá, Colombia, 1995-1997. Washington, D.C.: Banco Interamericano de Desarrollo, SOC-127, 2001. Disponible en: http://iadb.org/sds/soc Acceso el 11 de agosto 2005.

MOCKUS, A. Armonizar ley, moraly cultura. Cultura ciudadana, prioridad de gobierno con resultados en prevención y control de violencia en Bogotá, 1995-1997, 2002a. Disponible en: http://www.iadb.org/sds/doc/2104spa.pdf Acceso el: 11 ago 2005.

MOCKUS, A. Convivencia como armonización de ley, moral y cultura. Perspectivas, v. XXXII, n. 1, p. 19-37, 2002b.

MOORE, $H$. The problem of explaining violence in the social sciences. In: HARVEY, P. y GOW (Ed.). Sex and violence: issues in representation and experience. Londres: Routledge, 1994. p. 138-155.

MOYANO DÍAZ, E.; GUAJARDO ESPINOZA, A.; VARGAS ARAVENA, J. Exploración del discurso acerca de la seguridad de tránsito en usuarios de vías en Santiago de Chile. In: Psicología del tráfico y la seguridad vial. Simposio y mesas temáticas, 1999. Disponible en: http://copsa.cop.es/congresoiberoa/base/franco/14.num Acceso el: 12 oct 1999.

O’ DONNELL, G. Situaciones: Micro-escenas de la privatización de lo público en Sao Paulo. Working Paper 121, Kellog Institute, 1989.

PELTZER, R. Motociclistas jóvenes en la ciudad de Mar del Plata. In: GELDSTEIN, R.; BERTONCELLO, R. (Coord.) Aspectos demográficos y sociales de los accidentes de tránsito en áreas seleccionadas de la Argentina. Buenos Aires: Ministerio de Salud y Ambiente de la Nación, 2006 p. 117- 136.

PETERLINI, C.; GELDSTEIN, R. El tránsito y sus accidentes. Estructura y agencia en las perspectivas de automovilistas y peatones de la Ciudad de Buenos Aires. In: ABRAMZÓN, M. et al. (Ed..) VI Jornadas Nacionales de debate interdisciplinario en salud y población. Buenos Aires: Instituto Gino Germani, 2005 (Eventos Académicos n. 3).

REY, C. Ciclistas adolescentes y jóvenes en la ciudad de Resistencia. In: GELDSTEIN, R.; BERTONCELLO, R. (Coord.) Aspectos demográficos y sociales de los accidentes de tránsito en áreas seleccionadas de la Argentina. Buenos Aires: Ministerio de Salud y Ambiente de la Nación, 2006. p. 137-150. 

Press, 1989.

VASILACHIS DE GIALDINO, I. La investigación cualitativa. In: VASILACHIS DE GIALDINO, I. (Coord.). Estrategias de investigación cualitativa. Buenos Aires: Gedisa, 2007. p. 23-64.

WEBER, M. La objetividad cognoscitiva de la ciencia social y de la política social. In: WEBER, M. Ensayos sobre metodología sociológica. Buenos Aires: Amorrortu, 1993.

WRIGHT, P.; MOREIRA, M.V.; SOICH, D. Antropología vial: símbolos, metáforas, y prácticas en el juego de la calle de conductores y peatones en Buenos Aires. In: Seminario del Centro de Investigaciones Etnográficas, Universidad Nacional de San Martin, 2007. mimeo.

\section{Notas}

${ }^{1}$ Entre 2000 y 2004 se registraron un poco más de 17 mil muertes por AT. Según las estadísticas vitales, el $41 \%$ de las víctimas fatales por AT se encontraba dentro de un automóvil. A partir del cambio de codificación de las causas de muerte en 1997 se puede analizar la mortalidad por accidentes de tránsito, según tipo de víctima en función del vehículo. Aquí se presentan datos del quinquenio 2000 04 debido a que es el último período del cual se dispone de la base de datos desagregada por causas. Sin embargo, un elevado porcentaje de los registros, el $28 \%$, no tiene identificado el tipo de transporte o vehículo, por lo que esta información debe analizarse con cautela (ALTIERI, 2008, p. 37).

${ }^{2}$ La elevada incidencia en los jóvenes y en los varones es una característica que observamos en las muertes por causas violentas (homicidios y suicidios) en general, y que no sólo se dan en la Argentina, sino en la mayoría de los países del mundo (ALTIERI, 2008, p. 36).

${ }^{3}$ El carácter de agentes sociales de los propios investigadores constituye una amenaza latente contra la pretensión de objetividad toda vez que su interés científico se vuelca al estudio de situaciones de la vida cotidiana en los mismos contextos sociales en los que ellos interactúan como ciudadanos comunes. Es con la intención de minimizar los posibles sesgos debidos a esta circunstancia, que la reflexión acerca del punto de vista del investigador, de nuestra propia posición de sujeto frente al objeto de estudio y el examen honesto de nuestros posibles preconceptos, constituyen hoy en día un requisito de las buenas prácticas de investigación - de manera especial aunque no excluyente de la práctica de la investigación cualitativa-. La explicitación, por tanto, de nuestro carácter de agentes en el campo social del tránsito de Buenos Aires es un modo de informar al lector que, durante el proceso de investigación, hemos tenido presente dicha circunstancia y las amenazas que ella entraña. Más específicamente, nuestras variadas experiencias en ese campo (como mujeres y varones residentes en Buenos Aires, pertenecientes a diferentes generaciones, algunos/as de los/las cuales conducimos automóvil y/o bicicleta y otros/ as no lo hacemos, pero inevitablemente todos y todas somos también peatones/as, en las mismas calles que constituyen el escenario de las acciones y las interacciones que intentamos analizar) han contribuido en la etapa de formulación de preguntas de investigación acerca de las posibles relaciones entre género, generación y prácticas de conducción. No realizamos observación-participante sistemática como parte del trabajo de campo y, por lo tanto, nuestras experiencias personales en el campo social del tránsito no fueron consideradas para su inclusión sistemática en el proceso analítico. Y, puesto que seguimos los lineamientos de la teoría fundamentada (Grounded Theory), tratamos en general de no aplicar categorías analíticas a priori. Mediante la reflexividad y la intersubjetividad - que pusimos 
en práctica en las discusiones de equipo- intentamos controlar los posibles sesgos que nuestras experiencias personales podrían introducir en la interpretación de los resultados y categorías emergentes.

${ }^{4}$ Para una revisión de los aportes actuales de las ciencias sociales a las percepciones subjetivas del riesgo, haciendo hincapié en los vínculos entre jóvenes y normas en el tránsito, ver Mendes Diz (2007).

5 "Los habitus son sistemas de disposiciones duraderas y transferibles, estructuras estructuradas predispuestas a funcionar como estructuras estructurantes, es decir como principios generadores y organizadores de prácticas y de representaciones que pueden estar objetivamente adaptadas a su fin sin suponer la búsqueda consciente de fines ni el dominio expreso de las operaciones necesarias para alcanzarlos, objetivamente 'regladas' y 'regulares' sin ser en nada el producto de la obediencia a reglas y, siendo todo esto, colectivamente orquestadas sin ser el producto de la acción organizadora de un director de orquesta" (BOURDIEU, 1991, p. 88-89).

${ }^{6}$ Esta autora destaca la concepción del sujeto del post-post estructuralismo feminista, como individualidad que escapa a los moldes estrechos del género concebido como dos categorías opuestas y mutuamente excluyentes, sugiriendo que las características atribuidas a la categoría mujer o la categoría hombre serían más bien cualidades ("femeninas" o "masculinas") que, en diferente grado y composición, integrarían las subjetividades de los individuos concretos.

${ }^{7}$ Los estudios fueron realizados con el apoyo del Ministerio de Salud de la Nación (GELDSTEIN; BERTONCELLO, 2006; GELDSTEIN, 2008). Se realizó una muestra intencional, incluyendo un número similar de mujeres y de hombres con diferentes edades y niveles de educación formal. Las entrevistas fueron objeto de un primer análisis caso por caso. Los datos fueron ingresados luego a una base Atlas.ti para facilitar la clasificación y el análisis progresivo. Se aplicaron procedimientos clasificatorios y analíticos tomados de la "teoría fundamentada" (grounded theory) (GLASER; STRAUSS 1967, STRAUSS, 1989). Utilizamos la comparación constante como método básico de descubrimiento y construcción de categorías y la intersubjetividad y la reflexividad como formas de producción de conocimiento y como resguardo frente a las amenazas de validez (MAXWELL, 1996), teniendo presente la existencia de una doble o triple hermenéutica (VASILACHIS DE GIALDINO, 2007). Buscando aplicar los criterios de parsimonia - maximizar la comprensión de un fenómeno con el mínimo posible de conceptos - y de alcance - ampliar el campo de aplicación del análisis sin desligarse de la base empírica, fuimos identificando y analizando una serie de categorías centrales surgidas de los discursos de los sujetos.

${ }^{8}$ En la presentación de los resultados, empleamos la itálica para citar los "términos nativos", utilizados por los sujetos entrevistados.

${ }^{9}$ Ejemplos de la imposición de prácticas trasgresoras por parte de conductores agresivos y prepotentes son: hacer zig-zag, meterse en el espacio disponible entre auto y auto, pegarse al auto de adelante, apurar a los otros conductores, tocar bocina cuando no corresponde.

${ }^{10}$ Moore (1994) advierte que la violencia está siempre teñida de significaciones de género, que es sexuada, (engendered) independientemente del sexo de los actores individuales en situaciones concretas.

${ }^{11}$ La que, según este autor, explicaría lo que él denomina como la paradoja de la doxa.

${ }^{12}$ Aquí retomaremos parte de los recientes análisis de Mendes Diz (2007).

${ }^{13}$ Ver datos en Introducción. 
Gender, violence and risk in the road traffic. Male domination in the discourses of car drivers in Buenos Aires city

We present partial results from two Multicentric Studies on sociocultural and demographic aspects of traffic accidents in Argentina, conducted between 2004 and 2008 with financial support from the Ministry of Health. This paper focuses on the relationships between gender dimensions and driving habitus that emerged from 92 indepth interviews applied to an intentional sample of male and female non-professional car drivers in the Buenos Aires City. Qualitative analysis was performed following the interpretive paradigm. Grounded Theory procedures were applied and constant comparison was used for the discovery and construction of categories. We were able to identify the presence of the male domination phenomenon in the habitus and doxa (Bourdieu) of different categories of car drivers. Criticisms of feminine driving styles and discriminatory attitudes against female drivers were found in the dominant discourses of young males and of females of all ages. Different interviewed women value the male pragmatism in driving; while mature women consider it as a "natural" masculine attribute, young women adopt it in their own driving practices as a mean to avoid gender stigmatization. However, such a pragmatism is opposed to traffic legal rules and due respect towards other people in the public space and, as long as young women take masculine driving style as their model, the risk of traffic injuries would surely increase. Policies aimed at improving road safety should emphasize the kind of positive values of responsible driving that we discovered in the sub-dominant (feminine, mature) discourses that reminded us Gilligan's concept of the ethics of care.

> Key words: traffic accidents; gender violence; car drivers. 